\title{
Hepatoprotective activity against acetaminophen-induced liver dysfunction and GC-MS profiling of a brown algae Sargassum ilicifolium
}

Khan Hira ${ }^{1}$, Hafiza Farhat ${ }^{2}$, Nida Sohail ${ }^{3}$, Madeeha Ansari ${ }^{4}$, Jehan Ara $^{5}$ and Syed Ehteshamul-Haque ${ }^{2^{*}}$

\begin{abstract}
Background: Drug-induced hepatotoxicity is one of the most important causes of liver dysfunction. Acetaminophen (paracetamol) an analgesic-antipyretic drug is generally considered safe but its overdose may cause liver toxicity. Marine macro-algae (seaweeds) especially brown seaweeds possess unique biological activities including hepatoprotective potential. The current study focused on the hepatoprotective effect of different solvent fractions of Sargassum ilicifolium and characterization of its $n$-hexane soluble fraction.

Methods: The ethanol extract $(20 \mathrm{~g})$ of S. ilicifolium was mixed with solvents of increasing polarity, starting with $n$ hexane followed by chloroform and methanol. All three ( $n$-hexane, chloroform and methanol) soluble fractions were administered to the rats at dose of $150 \mathrm{mg} / \mathrm{kg}$, b.w. Intraperitoneal administration of acetaminophen (600 mg/ $\mathrm{kg}$ b.w.) to rats was used to cause liver injury. The hepatic damage was evaluated by liver markers enzymes; aspartate aminotransferases (AST), alanine aminotransferases (ALT), alkaline phosphatase (ALP), lactate dehydrogenase $(\mathrm{LDH})$, bilirubin along with other metabolites i.e., triglycerides, cholesterol, urea, glucose and creatinine. Lipid peroxidation and glutathione and were estimated in liver tissue. $n$-Hexane fraction was subjected to GC-MS analysis in order to identify potent compounds.
\end{abstract}

Results: The oral administration of $n$-hexane and methanol soluble fractions reduced the acetaminophenaugmented liver marker enzymes ALT, AST, ALP, LDH, along with bilirubin, urea, creatinine, glucose and triglycerides. The $n$-hexane and methanol soluble fractions also improved hepatic antioxidant level via enhancing hepatic glutathione and reversing lipid peroxidation. GC-MS spectroscopy of $n$-hexane fraction of S. ilicifolium revealed the presence of some new compounds. Among them, fatty acids were found to be in highest concentration followed by halogenated hydrocarbons, benzene derivatives, and sterols. Fatty acid in seaweed may be one of the factors for hepatoprotection from drug-induced hepatotoxicity.

Conclusion: From the results, it is evident that $n$-hexane and methanol soluble fractions of S. ilicifolium have the ability to protect the liver against toxicity, which is comparable with silymarin used as a standard drug. Sargassum ilicifolium contains bioactive compounds with pharmaceutical importance.

Keywords: Sargassum ilicifolium, Hepatoprotective activity, Drug-induced hepatotoxicity, n-hexane fraction, Silymarin, GC-MS

\footnotetext{
* Correspondence: sehaq@uok.edu.pk

${ }^{2}$ Department of Botany, Agricultural Biotechnology \& Phytopathology

Laboratory, University of Karachi, Karachi 75270, Pakistan

Full list of author information is available at the end of the article
}

(c) The Author(s). 2021 Open Access This article is licensed under a Creative Commons Attribution 4.0 International License which permits use, sharing, adaptation, distribution and reproduction in any medium or format, as long as you give appropriate credit to the original author(s) and the source, provide a link to the Creative Commons licence, and indicate if changes were made. The images or other third party material in this article are included in the article's Creative Commons licence, unless indicated otherwise in a credit line to the material. If material is not included in the article's Creative Commons licence and your intended use is not permitted by statutory regulation or exceeds the permitted use, you will need to obtain permission directly from the copyright holder. To view a copy of this licence, visit http://creativecommons.org/licenses/by/4.0/. 


\section{Introduction}

Acetaminophen (paracetamol) an analgesic-antipyretic drug is generally considered safe but its overdose may affect liver function [1-3] and causes severe hepatic necrosis to complete hepatic failure [4]. It also affects other organs and may trigger nephrotoxicity [5]. Since medicines used for managing liver diseases may have potential side effects thus progression of chronic liver diseases have not been prevented effectively with any therapy [6].

Marine macro-algae have shown great bioactivity potential including anti-inflammatory, antimicrobial, antiviral, antitumor, hypoglycemic and hypolipidemic activities [7-9], due to the presence of bioactive compounds that may be steroids, terpenoids, isoprenoids and sesquiterpenes [10]. The brown seaweeds, especially Sargassum species, are found in the shallow water of tropical and temperate regions [11-13] and contain a considerable amount of these bioactive compounds [14]. S. ilicifolium is one of the most commonly and abundantly found Sargassum species at Karachi coast. Several researchers evaluated the biological activities of S. ilicifolium [15-17]. Ambreen et al. [18] reported that S. ilicifolium contains calcium and ascorbic acid and also possesses antifungal activity. Similarly, Rebecca et al. [16] revealed antibacterial activity of ethanol extract of S. ilicifolium. The ethyl acetate fraction of S. ilicifolium has shown a prominent immune-stimulatory effect [15]. In our previous study, ethanol extract of S.ilicifolium (200 mg/kg b.w.) did not show any adverse effect on hepatic and renal function in rats $[2,5]$. Intraperitoneal administration of hexane, methanol or butanol extract of S. wightii did not cause mortality or showed toxic effect in mice [19]. The current report described the hepatoprotective role of solvent fractions and GC-MS profiling of $n$-hexane fraction of S.ilicifolium.

\section{Materials and methods Chemicals and reagents}

Acetaminophen, silymarin, polyethylene glycol, trichloroacetic acid (TCA),thiobarbituric acid, DTNB; 5, 5 dithio-bis-(2-nitrobenzoic acid) were purchased from Sigma Aldrich, U.S.A. Solvents (ethanol, butanol, chloroform, $n$-hexane and methanol) of analytical grades were purchased from Merck (France). All the kits including ALT, AST, ALP, LDH, bilirubin (total \& direct), glucose, triglycerides, cholesterol, urea and creatinine were purchased from Merck (France) and Ecoline (Germany).

\section{Collection of seaweed}

Sargassum ilicifolium was collected from Buleji beach, Karachi and washed under tap water in the laboratory, dried under shade and grinded to powder in an electric miller. The seaweed powder was stored at room temperature until use. Voucher specimen and herbarium sheet of the seaweed was prepared and kept in the Seaweed Herbarium (KUH-SW-891), MAH Qadri Biological Research Center, University of Karachi for record. Dr. Aisha Begum, Associate Professor, Department of Botany, University of Karachi, Karachi identified the seaweed.

\section{Ethanol extract and solvent fractions of S. ilicifolium}

Ethanol extract of $S$. ilicifolium was prepared by soaking $(500 \mathrm{~g})$, for 1 week in $2 \mathrm{~L}$ ethanol at room temperature. Then filtered over cotton wool and concentrated on a rotary vacuum evaporator (Buchi R-200 New Castle, DE) at $35^{\circ} \mathrm{C}$ to obtain a gummy mass that gives the yield of $4.8 \%$. A portion $(20 \mathrm{~g})$ of ethanol extract of seaweeds was mixed with $n$-hexane $(250 \mathrm{~mL})$ in a separating funnel. The $n$-hexane soluble portion was separated and concentrated on a rotary vacuum evaporator at $35^{\circ} \mathrm{C}$, that give an yield of $2.5 \%$. The $n$-hexane insoluble residue was extracted with chloroform, that give an yield of $6.5 \%$. The chloroform insoluble or remaining portion was mixed with methanol (91\%). All three ( $n$-hexane, chloroform and methanol) soluble fractions were stored in airtight vials at room temperature till used [20].

\section{Animals}

Male rats (140-170 g) of Wistar strain, obtained from Dow University of Health Sciences, Karachi were used in this study. They were kept in polyethylene cages, fed with standard pellet diet [21] and water. Animals were kept in the laboratory for 1 week to allow the animals to acclimatize with laboratory conditions. Experiments were conducted with the permission of Institutional Animal Research Ethical Committee.

\section{Induction of hepatotoxicity}

Acetaminophen (Sigma Aldrich, U.S.A.) was administered intraperitoneally at $600 \mathrm{mg} / \mathrm{kg}$, body weight (b.w.) and the dose was prepared in $40 \%$ polyethylene glycol (Sigma Aldrich, U.S.A.) with constant stirring and mild heating.

\section{Experimental design}

Rats were kept in pre bedded polyethylene cages (6 rats/ cage) with standard laboratory conditions (temperature $25 \pm 2{ }^{\circ} \mathrm{C}$ and $12 \mathrm{~h}$ light/dark cycle), fed with standard pellet diet prepared in the laboratory following the procedure of NRC (1995) [21] and tap water ad libitum. The animals were kept in the laboratory for 1 week before starting the experiment to acclimatize animals with laboratory conditions. Experiments were conducted with the permission of the Institutional Animal Research Ethical Committee. 


\section{Effect of solvent fractions of S. ilicifolium}

Rats were divided into six major groups and each group consisted of 6 rats to determine the effect of seaweed fractions in acetaminophen intoxicated rats.

\section{Normal control}

Rats were orally administered with distilled water $(1 \mathrm{ml} /$ $\mathrm{kg}$ b.w.) for 14 days along with the normal diet $(n=6)$.

\section{Sargassum ilicifolium fractions treated group}

This group was further divided into 3 subgroups viz.; I, II, III ( $\mathrm{n}=6$ in each sub-group) and rats were administered orally (p.o.) by solvent fractions (hexane, chloroform and methanol separately) of ethanol extract of the S. ilicifolium @ $150 \mathrm{mg} / \mathrm{kg}$ b.w., dissolved in water, daily for 14 days.

\section{Acetaminophen (AAP) control group}

Rats were administered with distilled water (p.o., $1 \mathrm{ml} / \mathrm{kg}$ b.w.) for 14 days along with the normal diet. On day 14 rats were intoxicated by a single intraperitoneal injection of acetaminophen (i.p., $600 \mathrm{mg} / \mathrm{kg}$ b.w., in saline).

\section{Sargassum ilicifolium fractions + acetaminophen treated group}

This group was further divided into 3 subgroups viz.; I, II, III. Each group $(n=6)$ was administered orally with $n$-hexane, chloroform and methanol fractions of $S$. ilicifolium at the dose of $150 \mathrm{mg} / \mathrm{kg}$ b.w., daily for 14 days. On day 14th rats were intoxicated by a single intraperitoneal injection of acetaminophen (i.p., $600 \mathrm{mg} / \mathrm{kg}$ b.w., in saline) in each subgroup.

\section{Silymarin treated group}

Treated with silymarin at the dose of $50 \mathrm{mg} / \mathrm{kg}$ b.w., suspended in distilled water and given orally to rats daily for 14 days.

\section{Silymarin treated group + acetaminophen treated group}

Treated with silymarin at the dose of $50 \mathrm{mg} / \mathrm{kg}$ daily for 14 days and injected with AAP on the last day.

On the 15th day all animals were decapitated after 12 $\mathrm{h}$ fasting and blood was collected for assessment of liver marker enzymes and other biochemical parameters.

\section{Assessment of hepatic damage}

Liver enzymes ALP, ALT, LDH, AST and metabolites bilirubin, creatinine, urea, triglycerides, cholesterol and glucose in serum were determined by using kits from Merck (France) and Ecoline (Germany). Glutathione and lipid peroxidation (MDA) in liver tissue was determined according to the method of Samarth et al. [22] and Ohkawa et al. [23] respectively.
Characterization of $n$-hexane fraction of S. ilicifolium by gas chromatography and mass spectrometry (GC-MS) $n$-Hexane fraction was subjected to GC-MS analysis on Agilent 6890 Gas Chromatograph hyphenated with Mass Spectrometer, Jeol, JMS- $600 \mathrm{H}$. The peak of each compound was identified by comparing their retention indices and mass spectra against National Institute of Standards and Technology (mainlib) USA and compared with Science finder [24].

\section{Statistical analysis}

Data were analyzed and their means were compared at a significant level $(p<0.05)$ using Duncan's multiple range test [25].

\section{Results}

Effect on serum biochemical markers

Acetaminophen intoxication significantly $(p<0.005)$ raised liver function markers like ALP, ALT, LDH, AST and bilirubin level in serum. $n$-Hexane fraction of $S$. ilicifolium showed improvement as evident by considerable decline of serum AST, ALT, ALP, LDH and bilirubin levels. The methanol soluble fraction also significantly $(p<0.005)$ reversed the increased level of ALP, ALT, LDH, AST and bilirubin. Chloroform soluble fraction was found less effective. The acetaminophen also affected kidney function and increased creatinine and urea many fold as compared to control. The creatinine and urea were decreased significantly in $n$-hexane and methanol fraction in comparison with AAP control. The glucose concentration was reached to (169.6) after acetaminophen intoxication and it was reduced up to ($22 \%)$ and $(-30.4 \%)$ in $n$-hexane and methanol fractions pretreated rats respectively. The cholesterol level was restored towards normal value in rats pretreated with $n$ hexane fraction $(+32.5 \%)$ of $S$. ilicifolium (Tables $1 \& 2$ ).

Administration of chloroform soluble fraction of S. ilicifolium to normal rats for 14 days did not influence the liver enzymes except for AST and LDH, while other liver metabolites like bilirubin, creatinine and urea remain unchanged when compared with normal rats. Similarly, $n$-hexane fraction did not alter the ALT, ALP, glucose level and other biochemical markers. The rats pretreated with a methanol fraction of S. ilicifolium also not shown any adverse effect on enzymes, but reduced glucose level than control rats. Creatinine, cholesterol and triglyceride level were found increased by the methanol fraction of $S$. ilicifolium (Tables 1 \& 2).

Administration of silymarin in AAP intoxicated rats significantly decreased the liver enzymes and bilirubin level as compared to AAP intoxicated rats. On the other side, kidney profile; creatinine and urea, were also reduced in silymarin + AAP treated group as compared to AAP control rats. Similarly, silymarin in AAP 
Table 1 Effect of $n$-hexane, chloroform and methanol soluble fractions of Sargassum ilicifolium on liver enzymes and bilirubin in normal and acetaminophen (AAP) dosed rats

\begin{tabular}{|c|c|c|c|c|c|c|c|c|c|c|}
\hline \multirow[t]{2}{*}{ Rat groups } & $\begin{array}{l}\text { Normal } \\
\text { rats }\end{array}$ & $\begin{array}{l}\text { AAP } \\
\text { dosed } \\
\text { rats }\end{array}$ & $\begin{array}{l}\text { Normal } \\
\text { rats }\end{array}$ & $\begin{array}{l}\text { AAP } \\
\text { dosed rats }\end{array}$ & $\begin{array}{l}\text { Normal } \\
\text { rats }\end{array}$ & $\begin{array}{l}\text { AAP } \\
\text { dosed rats }\end{array}$ & $\begin{array}{l}\text { Normal } \\
\text { rats }\end{array}$ & $\begin{array}{l}\text { AAP dosed } \\
\text { rats }\end{array}$ & $\begin{array}{l}\text { Normal } \\
\text { rats }\end{array}$ & $\begin{array}{l}\text { AAP } \\
\text { dosed rats }\end{array}$ \\
\hline & \multicolumn{2}{|l|}{ ALT (U/L) } & \multicolumn{2}{|l|}{ AST (U/L) } & \multicolumn{2}{|l|}{ ALP (U/L) } & \multicolumn{2}{|l|}{ LDH (U/L) } & \multicolumn{2}{|c|}{ Bilirubin (mg/dl) } \\
\hline Control & $\begin{array}{l}33.6^{\mathrm{ab}} \pm \\
3.5^{2}\end{array}$ & $\begin{array}{l}104.6^{\mathrm{a}} \pm \\
9.4\end{array}$ & $134.3^{c} \pm 6$ & $\begin{array}{l}239.3^{b} \pm \\
8.1\end{array}$ & $\begin{array}{l}67.6^{\mathrm{a}} \pm \\
6.5\end{array}$ & $\begin{array}{l}113.6^{\mathrm{a}} \pm \\
10.4\end{array}$ & $328.6^{a} \pm 10$ & $396.3^{a} \pm 6$ & $0.4^{a} \pm 0$ & $0.86^{\mathrm{a}} \pm 0.06$ \\
\hline $\begin{array}{l}\text { hexane fraction of } \\
\text { S. ilicifolium }\end{array}$ & $\begin{array}{l}30.3^{b} \pm 2.3 \\
(-9.8 \%)\end{array}$ & $\begin{array}{l}63^{b} \pm 2.6 \\
(-39.7 \%)\end{array}$ & $\begin{array}{l}166.3^{b} \pm 8.6 \\
(+23.8 \%)\end{array}$ & $\begin{array}{l}178.3^{c} \pm 9.6 \\
(-25.4 \%)\end{array}$ & $\begin{array}{l}74^{a} \pm 3 \\
(+9.4 \%)\end{array}$ & $\begin{array}{l}87.3^{b} \pm 2.1 \\
(-23.1 \%)\end{array}$ & $\begin{array}{l}312.6^{\mathrm{b}} \pm \\
4.5(- \\
4.8 \%)\end{array}$ & $\begin{array}{l}303.3^{b} \pm 22 \\
(-23.4 \%)\end{array}$ & $\begin{array}{l}0.46^{\mathrm{a}} \pm 0.1 \\
(+15 \%)\end{array}$ & $\begin{array}{l}0.6^{b} \pm 0.1 \\
(-30.2 \%)\end{array}$ \\
\hline $\begin{array}{l}\text { Chloroform } \\
\text { fraction of } S \text {. } \\
\text { ilicifolium }\end{array}$ & $\begin{array}{l}38.3^{\mathrm{a}} \pm 3.5 \\
(+13.9 \%)\end{array}$ & $\begin{array}{l}99.6^{\mathrm{a}} \pm 17 \\
(-4.7 \%)\end{array}$ & $\begin{array}{l}237.6^{\mathrm{a}} \pm \\
19.4(+ \\
76.9 \%)\end{array}$ & $\begin{array}{l}295.3^{a} \pm 8 \\
(+23.4 \%)\end{array}$ & $\begin{array}{l}67.6^{\mathrm{a} \pm} \\
3.5(0)\end{array}$ & $\begin{array}{l}106.6^{a} \pm 6.5 \\
(-6.16 \%)\end{array}$ & $\begin{array}{l}298^{b} \pm 7 \\
(-9.3 \%)\end{array}$ & $\begin{array}{l}303^{b} \pm 8 \\
(-23.5 \%)\end{array}$ & $\begin{array}{l}0.43^{\mathrm{a}} \pm \\
0.05(+ \\
7.5 \%)\end{array}$ & $\begin{array}{l}0.66^{b} \pm 0.06 \\
(-23.2 \%)\end{array}$ \\
\hline $\begin{array}{l}\text { Methanol fraction } \\
\text { of S. ilicifolium }\end{array}$ & $\begin{array}{l}35.6^{\mathrm{ab}} \pm \\
3.2(+ \\
5.9 \%)\end{array}$ & $\begin{array}{l}66.6^{b} \pm 2.5 \\
(-36.3 \%)\end{array}$ & $\begin{array}{l}180.3^{b} \pm 4.5 \\
(+34.2 \%)\end{array}$ & $\begin{array}{l}182.3^{c} \pm 13 \\
(-23.8 \%)\end{array}$ & $\begin{array}{l}71^{\mathrm{a}} \pm 2.6 \\
(+5.0 \%)\end{array}$ & $\begin{array}{l}79.6^{\mathrm{b}} \pm 2 \\
(-29.9 \%)\end{array}$ & $\begin{array}{l}312.6^{\mathrm{b}} \pm 9 \\
(-4.86 \%)\end{array}$ & $\begin{array}{l}331.3^{b} \pm \\
19.6(- \\
16.4 \%)\end{array}$ & $\begin{array}{l}0.46^{\mathrm{a}} \pm 0 \\
(+15 \%)\end{array}$ & $\begin{array}{l}0.53^{b} \pm 0.06 \\
(-38.3 \%)\end{array}$ \\
\hline
\end{tabular}

The values were expressed as means \pm Standard error $(n=6)$. The values having the same superscript within the column are not significantly $(p<0.05)$ different according to Duncan's multiple range test. Values in parenthesis are showing percent increased or decreased as compared to their respective control

intoxicated rats also showed protective effect by lowering the glucose level and lipid parameters (cholesterol and triglyceride) as compared to AAP control group (Tables 3 \& 4).

\section{Effect on solvent fractions of S. ilicifolium on glutathione (GSH) and lipid peroxidation (TBARS)}

Glutathione $(\mathrm{GSH})$ level was significantly $(p<0.05)$ decreased in acetaminophen (AAP) treated rats as compared to normal control rats. However, glutathione level was improved and increased in rats pretreated with $n$ hexane, methanol and chloroform fractions of S. ilicifolium in acetaminophen intoxicated rats as compared to acetaminophen (AAP) control group. Methanol fraction improved this level and brought almost to the normal control rats, while $n$-hexane fraction increased this level higher than the normal control (Fig. 1).

AAP intoxicated rats significantly elevated TBARS [6.16 $\mu \mathrm{mole} / \mathrm{gm}$ wet liver tissue (w.l.t)] level as compared to normal control (2.4 $\mu$ mole/gm). n-hexane (3.19 $\mu \mathrm{mole} / \mathrm{gm})$ and methanol $(4.1 \mu \mathrm{mole} / \mathrm{gm})$ fraction of S. ilicifolium significantly reduced TBARS level as compared to AAP intoxicated rats (Fig. 1). The overall highest hepatoprotective activity was found in $n$-hexane soluble fraction of $S$. ilicifolium followed by methanol fraction. The $n$-hexane fraction was thus characterized on GC-MS considering its hepatoprotective activity.

Glutathione level was significantly improved and TBARS level decreased in rats treated with silymarin $(50 \mathrm{mg} / \mathrm{kg}$ b.w.) in acetaminophen intoxicated rats as

Table 2 Effect of $n$-hexane, chloroform and methanol soluble fractions of Sargassum ilicifolium on glucose, lipid parameters and kidney function markers in normal and acetaminophen dosed rats

\begin{tabular}{|c|c|c|c|c|c|c|c|c|c|c|}
\hline \multirow[t]{2}{*}{ Rat groups } & $\begin{array}{l}\text { Normal } \\
\text { rats }\end{array}$ & $\begin{array}{l}\text { AAP } \\
\text { dosed rats }\end{array}$ & $\begin{array}{l}\text { Normal } \\
\text { rats }\end{array}$ & $\begin{array}{l}\text { AAP } \\
\text { dosed rats }\end{array}$ & $\begin{array}{l}\text { Normal } \\
\text { rats }\end{array}$ & $\begin{array}{l}\text { AAP } \\
\text { dosed } \\
\text { rats }\end{array}$ & $\begin{array}{l}\text { Normal } \\
\text { Rats }\end{array}$ & $\begin{array}{l}\text { AAP } \\
\text { dosed } \\
\text { rats }\end{array}$ & $\begin{array}{l}\text { Normal } \\
\text { rats }\end{array}$ & $\begin{array}{l}\text { AAP dosed } \\
\text { rats }\end{array}$ \\
\hline & \multicolumn{2}{|c|}{ Glucose (mg/dl) } & \multicolumn{2}{|c|}{ Triglyceride (mg/dl) } & \multicolumn{2}{|c|}{ Cholesterol (mg/dl) } & \multicolumn{2}{|c|}{ Urea (mg/dl) } & \multicolumn{2}{|c|}{ Creatinine (mg/dl) } \\
\hline Control & $83.6^{a} \pm 4.7$ & $169.6^{a} \pm 8.7$ & $74.3^{b} \pm 5.1$ & $\begin{array}{l}104.3^{b} \pm \\
1.5\end{array}$ & $73.6^{b} \pm 6.8$ & $\begin{array}{l}55.6^{\mathrm{b}} \pm \\
2.08\end{array}$ & $26.3^{a} \pm 2.5$ & $42^{a} \pm 2$ & $0.83^{b} \pm 0.06$ & $1.3^{\mathrm{a}} \pm 0.1$ \\
\hline $\begin{array}{l}\text { hexane fraction of } \\
\text { S. ilicifolium }\end{array}$ & $\begin{array}{l}81^{a} \pm 5.5 \\
(-3.11 \%)\end{array}$ & $\begin{array}{l}131.3^{b} \pm \\
3.2(- \\
22.5 \%)\end{array}$ & $\begin{array}{l}75.6^{\mathrm{b}} \pm 4.9 \\
(+1.7 \%)\end{array}$ & $\begin{array}{l}110.3^{b} \pm 4 \\
(+5.75 \%)\end{array}$ & $\begin{array}{l}75.6^{b} \pm 6.4 \\
(+2.71 \%)\end{array}$ & $\begin{array}{l}73.6^{\mathrm{a}} \pm 2.8 \\
(+32.3 \%)\end{array}$ & $\begin{array}{l}25.6^{\mathrm{a}} \pm 1.5 \\
(-2.66 \%)\end{array}$ & $\begin{array}{l}29^{b} \pm 2.6 \\
(-30.9 \%)\end{array}$ & $\begin{array}{l}0.96^{\mathrm{a}} \pm 0.06 \\
(+15.6 \%)\end{array}$ & $\begin{array}{l}0.9^{b} \pm 0.1 \\
(-30.7 \%)\end{array}$ \\
\hline $\begin{array}{l}\text { Chloroform } \\
\text { fraction of S. } \\
\text { ilicifolium }\end{array}$ & $\begin{array}{l}71.6^{b} \pm 3.7 \\
(-14.3 \%)\end{array}$ & $\begin{array}{l}154.3^{\mathrm{a}} \pm \\
15.6(- \\
9.0 \%)\end{array}$ & $\begin{array}{l}91.6^{\mathrm{a}} \pm 3.7 \\
(+23.2 \%)\end{array}$ & $\begin{array}{l}109.6^{b} \pm \\
4.6(+ \\
5.08 \%)\end{array}$ & $\begin{array}{l}81.3^{b} \pm 3.2 \\
(+10.4 \%)\end{array}$ & $\begin{array}{l}56.3^{b} \pm 5.5 \\
(+1.25 \%)\end{array}$ & $\begin{array}{l}28.6^{\mathrm{a}} \pm \\
1.15(+ \\
8.7 \%)\end{array}$ & $\begin{array}{l}39^{a} \pm 1 \\
(-7.14 \%)\end{array}$ & $\begin{array}{l}0.92^{\mathrm{ab}} \pm \\
0.05(+ \\
10.8 \%)\end{array}$ & $\begin{array}{l}1.23^{\mathrm{a}} \pm 0.06 \\
(-5.38 \%)\end{array}$ \\
\hline $\begin{array}{l}\text { Methanol fraction } \\
\text { of S. ilicifolium }\end{array}$ & $\begin{array}{l}63^{c} \pm 3.6 \\
(-24.6 \%)\end{array}$ & $\begin{array}{l}118^{b} \pm 5.2 \\
(-30.4 \%)\end{array}$ & $\begin{array}{l}87.6^{a b} \pm \\
11.06(+ \\
17.9 \%)\end{array}$ & $\begin{array}{l}123^{a} \pm 5 \\
(+17.9 \%)\end{array}$ & $\begin{array}{l}92.3^{\mathrm{a}} \pm 3.2 \\
(+25.4 \%)\end{array}$ & $\begin{array}{l}68.3^{\mathrm{a}} \pm 4.6 \\
(+22.8 \%)\end{array}$ & $\begin{array}{l}26^{a} \pm 2.6 \\
(-1.14 \%)\end{array}$ & $\begin{array}{l}25^{\mathrm{C}} \pm 2 \\
(-40.4 \%)\end{array}$ & $\begin{array}{l}1.03^{\mathrm{a}} \pm 0.05 \\
(+24.0 \%)\end{array}$ & $\begin{array}{l}0.96^{b} \pm 0.06 \\
(-26.15 \%)\end{array}$ \\
\hline
\end{tabular}

The values were expressed as means \pm Standard error $(n=6)$

The values having the same superscript within the column are not significantly $(p<0.05)$ different according to Duncan's multiple range test

Values in parenthesis are showing percent increased or decreased as compared to their respective control 
Table 3 Effect of silymarin at dose of $50 \mathrm{mg} / \mathrm{kg}$ b.w., on liver enzymes and bilirubin in normal and acetaminophen (AAP) dosed rats

\begin{tabular}{llllll}
\hline Rat groups & ALT (U/L) & AST (U/L) & ALP (U/L) & LDH (U/L) & Bilirubin (mg/dl) \\
\hline Normal control & $35.6^{\mathrm{b}} \pm 2.08$ & $94.6^{\mathrm{c}} \pm 6.8$ & $53^{\mathrm{c}} \pm 7$ & $216.3^{\mathrm{c}} \pm 13.05$ & $0.56^{\mathrm{c}} \pm 0.057$ \\
Silymarin (50 mg/kg b.w.) & $34.3^{\mathrm{b}} \pm 3.5^{\mathrm{a}}(-3.6 \%)$ & $91.6^{\mathrm{c}} \pm 14.5^{\mathrm{a}}(-3.1 \%)$ & $43.6^{\mathrm{c}} \pm 4.7^{\mathrm{a}}(-$ & $176.3^{\mathrm{d}} \pm 20.1^{\mathrm{a}}(-$ & $0.46^{\mathrm{c}} \pm 0.115^{\mathrm{a}}(-$ \\
& & & $17.7 \%)$ & $18.4 \%)$ & $17.8 \%)$ \\
Acetaminophen & $91.3^{\mathrm{a}} \pm 10.9^{\mathrm{a}}(+$ & $214.6^{\mathrm{a}} \pm 20.5^{\mathrm{a}}(+$ & $112^{\mathrm{a}} \pm 13.5^{\mathrm{a}}(+$ & $374^{\mathrm{a}} \pm 11.1^{\mathrm{a}}(+$ & $0.9^{\mathrm{a}} \pm 0.1^{\mathrm{a}}(+60.7 \%)$ \\
& $156.4 \%)$ & $126.8 \%)$ & $111.3 \%)$ & $72.9 \%)$ & \\
Silymarin (50 mg/ kg b.w.) & $44.3^{\mathrm{b}} \pm 6.8^{\mathrm{b}}(-$ & $120.6^{\mathrm{b}} \pm 8.3^{\mathrm{b}}(-$ & $79^{\mathrm{b}} \pm 5.2^{\mathrm{b}}(-29.4 \%)$ & $250.6^{\mathrm{b}} \pm 10.2^{\mathrm{b}}(-$ & $0.73^{\mathrm{b}} \pm 0.057^{\mathrm{b}}(-$ \\
Acetaminophen & $51.4 \%)$ & $43.8 \%)$ & & $32.9 \%)$ & $18.8 \%)$ \\
\hline
\end{tabular}

The values were expressed as means \pm standard error $(n=6)$

a Compared with normal control,

b Compared with acetaminophen control

Values in parenthesis are showing percent increased or decreased as compared to their respective control

The values having the same superscript within the column are not significantly $(p<0.05)$ different according to Duncan's multiple range test

compared to acetaminophen (AAP) control group (Fig. 2).

\section{GC/MS profiling of $\boldsymbol{n}$-hexane fraction of $S$. ilicifolium}

The GC/MS characterization of $n$-hexane soluble portion of S. ilicifolium revealed the presence of different volatile compounds like normal hydrocarbon, alcohols, fatty acid, aliphatic compounds, benzene derivatives, aldehyde and terpenoid. Total fifty one compounds were isolated and identified in which forty six compounds were found new from this source (Table 5). According to the results the highest concentration of hexadecanoic acid was found in our extract followed by octadecenoic acid. However the hexadecanoic is already a known compound from this source while octadecenoic acid is a new compound from $S$. ilicifolium. Our results showed the presence of steroids; spiro (1, 3-dioxolane)-2, 3'-(5'androsten-16'-ol) and estra-1, 3, 5(10)-trien-17 $\beta$-ol in $S$. ilicifolium (Table 5 \& Fig. 3; Fig. S- 1, S-2, S-3, S-4).

\section{Discussion}

In this study, pretreatment with $n$-hexane fractions of $S$. ilicifolium significantly decreased the toxin (acetaminophen) induced raised in serum transaminases, alkaline phosphatase, lactate dehydrogenase and bilirubin level. The methanol soluble fraction also reversed the increased level of ALT, AST, ALP and LDH but to a lower extent than $n$-hexane fraction. Raghavendran et al. [26] and Raghavendran and Srinivasan [27] have reported the protective role of ethanol and water extracts of $S$. polycystum against AAP induced hepatic damage. The drug induced hepatotoxicity may trigger nephrotoxicity as well [5]. An increase in urea and creatinine concentration in serum is indicative of nephrotoxicity [28]. In this study, adverse effect of acetaminophen was also found in AAP dosed rats, which was attenuated in rats pretreated with $n$-hexane fraction of S.ilicifolium evident from decreased concentration of urea and creatinine. Hepatotoxicity may affect lipid and glucose metabolism. In our study, $n$-hexane and methanol fractions of S. ilicifolium lowered the raised triglycerides level and improved decreased level of cholesterol in AAP dosed rats. Taj et al. [29] reported attenuation of adverse effect of AAP on liver and kidney function and glucose metabolism by the ethanol extract of a brown alga Stokeyia indica. Similarly Sohail et al. [5] reported the hepatoprotective and nephroprotective role of ethanol extract of S.ilicifolium in drug induced hepatotoxicity and nephrotoxicity in

Table 4 Effect of silymarin at dose of $50 \mathrm{mg} / \mathrm{kg}$ b.w., on glucose, lipid parameters and kidney function markers in normal and acetaminophen (AAP) dosed rats

\begin{tabular}{|c|c|c|c|c|c|}
\hline Groups & $\begin{array}{l}\text { Glucose } \\
\text { (mg/dl) }\end{array}$ & $\begin{array}{l}\text { Triglycerides } \\
(\mathrm{mg} / \mathrm{dl})\end{array}$ & $\begin{array}{l}\text { Cholesterol } \\
\text { (mg/dl) }\end{array}$ & $\begin{array}{l}\text { Urea } \\
\text { (mg/dl) }\end{array}$ & $\begin{array}{l}\text { Creatinine } \\
\text { (mg/dl) }\end{array}$ \\
\hline Normal control & $95^{c} \pm 7.5$ & $95^{b} \pm 6.2$ & $97^{\mathrm{a}} \pm 8.8$ & $31.6^{c} \pm 2.08$ & $0.90^{b} \pm 0.1$ \\
\hline Silymarin (50 mg/ kg b.w.) & $89^{c} \pm 5.2^{a}(-1.3 \%)$ & $82^{\mathrm{b}} \pm 6^{\mathrm{a}}(-8.6 \%)$ & $88^{\mathrm{a}} \pm 4.3^{\mathrm{a}}(-6.2 \%)$ & $\begin{array}{l}24.3^{\mathrm{d}} \pm 2.08^{\mathrm{a}}(- \\
45.2 \%)\end{array}$ & $\begin{array}{l}0.66^{\mathrm{c}} \pm 0.11^{\mathrm{a}}(- \\
\left.26.6^{\mathrm{a}}\right)\end{array}$ \\
\hline Acetaminophen & $\begin{array}{l}151^{\mathrm{a}} \pm 3.6^{\mathrm{a}}(+ \\
63.9 \%)\end{array}$ & $\begin{array}{l}133.6^{\mathrm{a}} \pm 9.5^{\mathrm{a}}(+ \\
45.6 \%)\end{array}$ & $70^{\mathrm{b}} \pm 8.7^{\mathrm{a}}(-24.8 \%)$ & $44^{\mathrm{a}} \pm 1.7^{\mathrm{a}}(+39.2 \%)$ & $1.3^{\mathrm{a}} \pm 0.1^{\mathrm{a}}(+44.4 \%)$ \\
\hline $\begin{array}{l}\text { Silymarin (50 mg/ kg b.w.) + } \\
\text { Acetaminophen }\end{array}$ & $\begin{array}{l}119.3^{b} \pm 5.6^{b}(- \\
71.9 \%)\end{array}$ & $\begin{array}{l}89.6^{b} \pm 8.7^{b}(- \\
66.5 \%)\end{array}$ & $\begin{array}{l}60.3^{b} \pm 2.08^{b}(- \\
16.1 \%)\end{array}$ & $\begin{array}{l}37.3^{b} \pm 2.08^{b}(- \\
15.2 \%)\end{array}$ & $\begin{array}{l}0.93^{b} \pm 0.15^{b}(- \\
28.4 \%)\end{array}$ \\
\hline
\end{tabular}

The values were expressed as means \pm standard error (ign $=6$ )

${ }^{a}$ Compared with normal control

${ }^{\mathrm{b}}$ Compared with acetaminophen control

Values in parenthesis are showing percent increased or decreased as compared to their respective control

The values having the same superscript within the column are not significantly $(p<0.05)$ different according to Duncan's multiple range test 


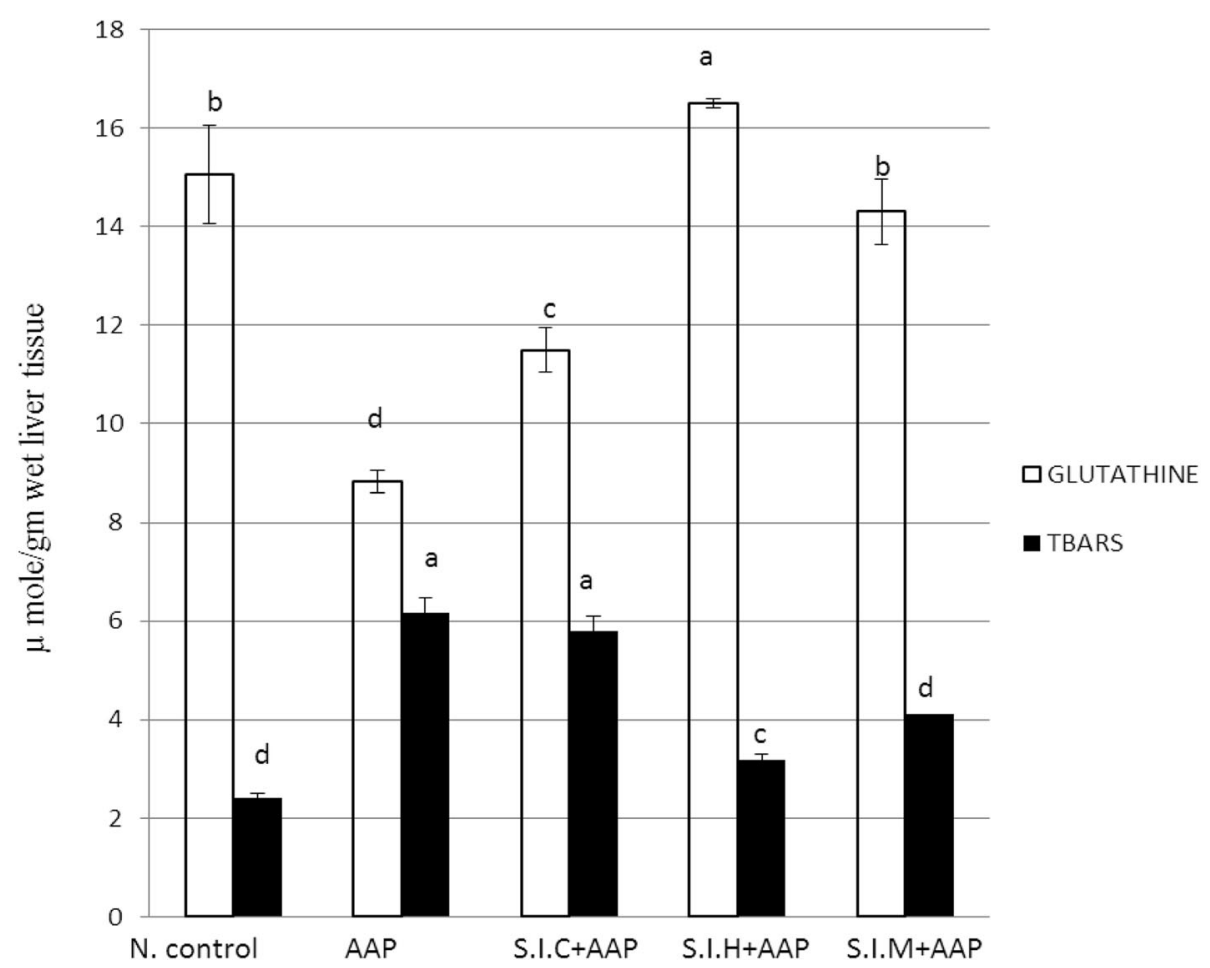

Fig. 1 Effect of chloroform, $n$-hexane and methanol soluble fractions of Sargassum ilicifolium on glutathione (GSH) and lipid peroxidation (TBARS) in acetaminophen (AAP) dosed rats. The bars in the graph showing mean \pm Standard error. The values having the same superscript on the bars are not significantly $(p<0.05)$ different according to Duncan's multiple range test

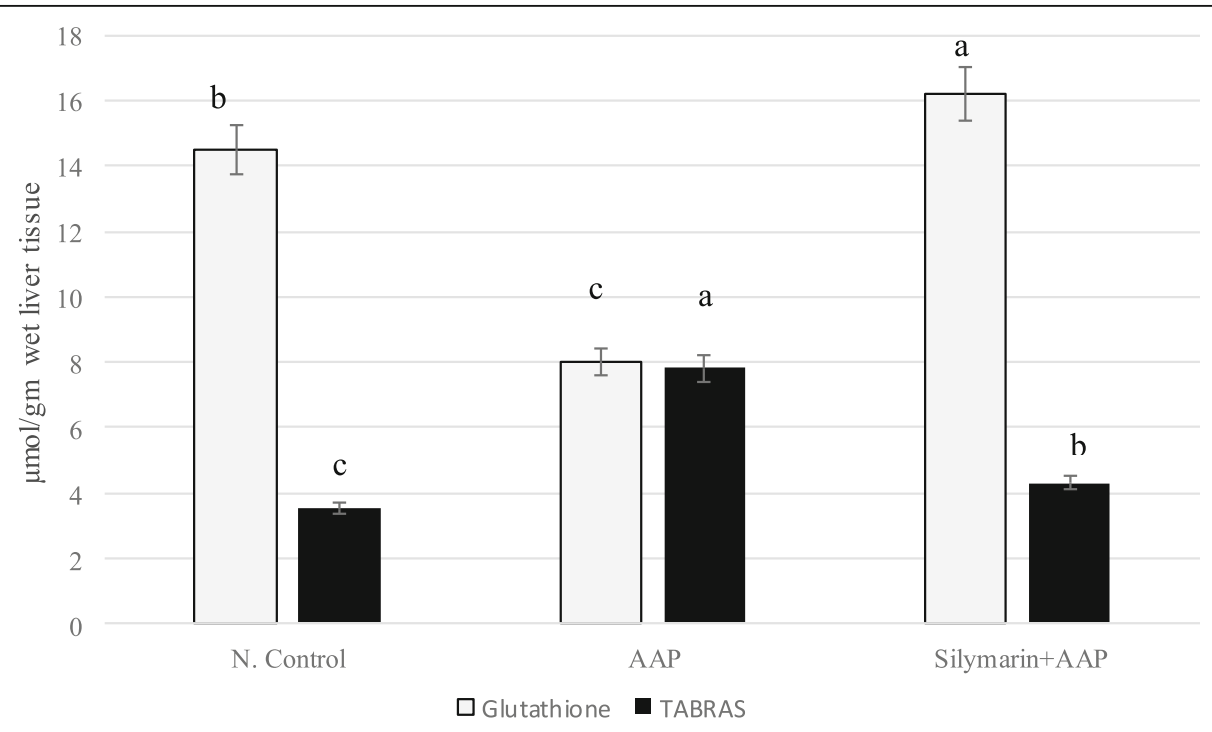

Fig. 2 Effect of silymarin at the dose of $50 \mathrm{mg} / \mathrm{kg}$ b.w., on glutathione and lipid peroxidation (TBARS) in acetaminophen dosed rats. The bars in the graph showing mean \pm Standard error $(n=6)$. The values bearing the same superscript on bar are not significantly $(p<0.05)$ different according to Duncan's multiple range test. $\mathrm{N}$. Control = Normal Control; AAP = Acetaminophen; SM = Silymarin 
Table 5 Spectral data of $n$-hexane soluble fraction of Sargassum ilicifolium

\begin{tabular}{|c|c|c|c|c|c|c|c|}
\hline $\begin{array}{l}\text { Peak } \\
\#\end{array}$ & Systemic name & $\begin{array}{l}\text { Molecular } \\
\text { formula }\end{array}$ & $\begin{array}{l}\text { Mol. } \\
\text { wt. }\end{array}$ & $\begin{array}{l}t_{\mathrm{R}} \\
(\min )\end{array}$ & Conc. & $\begin{array}{l}\text { Characteristic mass fragments, } \\
\mathrm{m} / \mathrm{z}(\text { rel.\% BP) }\end{array}$ & $\begin{array}{l}\text { Area } \\
\text { Sum } \\
(\%)\end{array}$ \\
\hline 1. & Methyl 3,3-dichloropropenoate & $\mathrm{C}_{4} \mathrm{H}_{4} \mathrm{Cl}_{2} \mathrm{O}_{2}$ & 154 & 6.104 & 0.092 & $\begin{array}{l}\text { 123(99), 125(66), 60(20), 63(15), 127(14), } \\
\text { 95(13), 154(99), 97(92), 156(77), 62(76) }\end{array}$ & 0.092 \\
\hline 2. & Benzaldehyde & $\mathrm{C}_{7} \mathrm{H}_{6} \mathrm{O}$ & 106 & 8.132 & 0.092 & $\begin{array}{l}\text { 106(99), 105(94), 77(92), 51(36), 50(18), } \\
78(16), 52(98), 107(77), 74(63), 39(60)\end{array}$ & 0.092 \\
\hline 3. & 3, 3, 5, 5-Tetramethylcyclohexanol & $\mathrm{C}_{10} \mathrm{H}_{20} \mathrm{O}$ & 156 & 10.201 & 0.16 & $\begin{array}{l}\text { 123(99), 41(44), 57(42), 81(29), 55(28), } \\
56(26), 85(25), 29(20), 43(19), 39(18)\end{array}$ & 0.16 \\
\hline 4. & Benzyl Alcohol & $\mathrm{C}_{7} \mathrm{H}_{8} \mathrm{O}$ & 108 & 11.457 & 0.242 & $\begin{array}{l}\text { 79(99), 108(89), 107(69), 77(54), 51(22), } \\
91(18), 78(11), 50(10), 39(10), 80(95)\end{array}$ & 0.242 \\
\hline 5. & 1-Propene, 2, 3-dibromo- & $\mathrm{C}_{3} \mathrm{H}_{4} \mathrm{Br}_{2}$ & 198 & 12.987 & 0.107 & $\begin{array}{l}119(99), 121(98), 39(90), 200(71), 198(37) \\
202(35), 40(30), 38(21), 37(13), 93(98)\end{array}$ & 0.107 \\
\hline 6. & I-(+)-Ascorbic acid 2,6-dihexadecanoate & $\mathrm{C}_{38} \mathrm{H}_{68} \mathrm{O}_{8}$ & 652 & 14.737 & 0.071 & $\begin{array}{l}57(99), 73(91), 43(84), 60(74), 71(63) \\
55(57), 69(44), 41(43), 129(43), 85(41)\end{array}$ & 0.071 \\
\hline 7. & 2-Oxepanone & $\mathrm{C}_{6} \mathrm{H}_{10} \mathrm{O}_{2}$ & 114 & 15.122 & 0.109 & $\begin{array}{l}42(99), 55(96), 41(49), 84(44), 56(30) \\
28(26), 27(22), 70(21), 39(20), 114(20)\end{array}$ & 0.109 \\
\hline 8. & Palmitic anhydride & $\mathrm{C}_{32} \mathrm{H}_{62} \mathrm{O}_{3}$ & 494 & 15.612 & 0.1 & $\begin{array}{l}98(99), 256(37), 55(36), 43(27), 57(27) \\
83(26), 73(25), 112(24), 84(23), 239(23)\end{array}$ & 0.1 \\
\hline 9. & Cyclopropane, 1, 1-dichloro-2-methyl-3-pentyl- & $\mathrm{C}_{9} \mathrm{H}_{16} \mathrm{Cl}_{2}$ & 194 & 16.419 & 0.086 & $\begin{array}{l}110(99), 41(97), 43(96), 39(77), 56(74) \\
112(65), 55(60), 42(48), 69(36), 65(30)\end{array}$ & 0.086 \\
\hline 10. & Cyclopropane, 1,1-dichloro-2,2-dimethyl- & $\mathrm{C}_{5} \mathrm{H}_{8} \mathrm{Cl}_{2}$ & 138 & 18.954 & 0.089 & $\begin{array}{l}\text { 103(99), 123(96), 41(63), 125(61), 87(59), } \\
\text { 67(43), 39(34), 105(33), 42(25), 89(24) }\end{array}$ & 0.089 \\
\hline 11. & 2-Hexanone, 6-(acetyloxy) & $\mathrm{C}_{8} \mathrm{H}_{14} \mathrm{O}_{3}$ & 158 & 19.297 & 0.14 & $\begin{array}{l}43(99), 98(17), 56(10), 55(70), 41(60) \\
101(60), 58(50), 61(50), 83(50), 42(30)\end{array}$ & 0.14 \\
\hline 12. & Bis-(2,2,6,6-tetramethyl,-4-piperidyl) amide oxalic acid & $\mathrm{C}_{20} \mathrm{H}_{38} \mathrm{~N}_{4} \mathrm{O}_{2}$ & 366 & 20.732 & 0.149 & $\begin{array}{l}\text { 124(99), 98(66), 77(56), 105(56), 58(44), } \\
42(39), 122(31), 41(21), 51(21), 141(17)\end{array}$ & 0.149 \\
\hline 13. & 2-Decenal, (E)- & $\mathrm{C}_{10} \mathrm{H}_{18} \mathrm{O}$ & 154 & 22.112 & 0.104 & $\begin{array}{l}41(99), 43(99), 55(71), 70(55), 29(53) \\
39(48), 27(45), 57(43), 83(34), 69(30)\end{array}$ & 0.104 \\
\hline 14. & Benzeneacetic acid, 4-tetradecyl ester & $\mathrm{C}_{22} \mathrm{H}_{36} \mathrm{O}_{2}$ & 332 & 24.939 & 0.116 & $\begin{array}{l}91(99), 57(59), 43(42), 41(37), 71(33) \\
55(30), 39(21), 85(20), 44(19), 196(17)\end{array}$ & 0.116 \\
\hline 15. & $5,5,10,10$-Tetrachlorotricyclo[7.1.0.0(4,6)]decane & $\mathrm{C}_{10} \mathrm{H}_{12} \mathrm{Cl}_{4}$ & 272 & 25.683 & 0.066 & $\begin{array}{l}\text { 109(99), } 91(90), 111(73), 65(67), 39(65) \\
77(61), 127(53), 51(46), 79(38), 101(36)\end{array}$ & 0.066 \\
\hline 16. & $\begin{array}{l}\text { 4-(3-Hydroxy-2,2,6-trimethyl-7-oxa-bicyclo[4.1.0]hept-1- } \\
\text { yl)-but-3-en-2-one }\end{array}$ & $\mathrm{C}_{13} \mathrm{H}_{20} \mathrm{O}_{3}$ & 224 & 26.612 & 0.187 & $\begin{array}{l}\text { 43(99), 123(97), 109(50), 41(19), 55(18), } \\
\text { 124(17), 165(16), 101(14), 125(12), 83(11) }\end{array}$ & 0.187 \\
\hline 17. & 1-Nonene, 2-ethyl-3-(methoxymethoxy) & $\mathrm{C}_{13} \mathrm{H}_{26} \mathrm{O}_{2}$ & 214 & 29.205 & 0.075 & $\begin{array}{l}\text { 45(99), 129(36), 83(15),55(10), 41(73), } \\
97(64), 69(63), 43(55), 70(49), 99(49)\end{array}$ & 0.075 \\
\hline 18. & $\begin{array}{l}\text { 2-(2-Chloromethylene-1-hydroxycyclohexyl) propionic } \\
\text { acid, methyl ester }\end{array}$ & $\mathrm{C}_{11} \mathrm{H}_{17} \mathrm{ClO}_{3}$ & 232 & 29.628 & 0.085 & $\begin{array}{l}\text { 145(99), 109(75), 81(45), 88(44), 57(35), } \\
147(35), 197(32), 79(19), 41(19), 91(19)\end{array}$ & 0.085 \\
\hline 19. & 1-Ethyl-1-(undec-10-enyloxy)-1-silacyclohexane & $\mathrm{C}_{18} \mathrm{H}_{36} \mathrm{OSi}$ & 296 & 30.614 & 0.072 & $\begin{array}{l}115(99), 41(85), 55(61), 45(54), 29(43), \\
87(41), 43(39), 27(33), 39(27), 267(26)\end{array}$ & 0.072 \\
\hline 20. & 1-Methyl-1-(2-tridecyl)oxy-1-silacyclopentane & $\mathrm{C}_{18} \mathrm{H}_{38} \mathrm{OSi}$ & 298 & 30.911 & 0.131 & $\begin{array}{l}\text { 143(99), 41(73), 43(69), 39(42), 55(39), } \\
99(36), 45(27), 57(25), 42(21), 69(17)\end{array}$ & 0.131 \\
\hline 21. & 1-Pentene, 1,1,5-trichloro- & $\mathrm{C}_{5} \mathrm{H}_{7} \mathrm{Cl}_{3}$ & 172 & 31.971 & 0.068 & $\begin{array}{l}\text { 65(99), } 101(90), 136(47), 109(46), 138(31), \\
111(30), 39(29), 103(28), 73(18), 96(15)\end{array}$ & 0.068 \\
\hline 22. & $\begin{array}{l}\text { 2-Oxa-7-thiatricyclo[4.4.0.0(3,8)]decan-4-ol, 4- } \\
\text { methylbenzenesulfonate, stereoisomer (1-a, 3- } a, 4-\beta, 6 \text { - } \\
\text { a, 8-a)- }\end{array}$ & $\mathrm{C}_{15} \mathrm{H}_{18} \mathrm{O}_{4} \mathrm{~S}_{2}$ & 326 & 33.222 & 0.074 & $\begin{array}{l}91(99), 97(83), 154(68), 113(55), 41(55), \\
81(52), 65(46), 326(46), 119(38), 45(36)\end{array}$ & 0.074 \\
\hline 23. & Octahydrobenzo[b]pyran, 4a-acetoxy-5,5,8a-trimethyl- & $\mathrm{C}_{14} \mathrm{H}_{24} \mathrm{O}_{3}$ & 240 & 33.496 & 0.077 & $\begin{array}{l}111(99), 124(85), 43(69), 69(49), 41(45), \\
180(44), 55(28), 97(23), 71(22), 96(22)\end{array}$ & 0.077 \\
\hline 24. & 1-Octyne, 3-(methoxymethoxy)- & $\mathrm{C}_{10} \mathrm{H}_{18} \mathrm{O}_{2}$ & 170 & 33.78 & 0.086 & $\begin{array}{l}\text { 45(99), 26(71), 99(56), 84(15), 41(14), } \\
67(10), 56(99), 55(97), 39(884), 43(82)\end{array}$ & 0.086 \\
\hline 25. & Spiro(1,3-dioxolane)-2,3'-(5'-androsten-16'-ol) & $\mathrm{C}_{21} \mathrm{H}_{32} \mathrm{O}_{3}$ & 332 & 33.974 & 0.091 & $\begin{array}{l}\text { 99(99), 55(13), 100(10), } 91(88), 79(59) \\
105(56), 41(51), 77(46), 86(43), 332(41)\end{array}$ & 0.091 \\
\hline 26. & Oleic Acid & $\mathrm{C}_{18} \mathrm{H}_{34} \mathrm{O}_{2}$ & 282 & 35.904 & 0.146 & 55(99), 41(60), 69(56), 57(47), 43(35), & 0.146 \\
\hline
\end{tabular}


Table 5 Spectral data of $n$-hexane soluble fraction of Sargassum ilicifolium (Continued)

\begin{tabular}{|c|c|c|c|c|c|c|c|}
\hline $\begin{array}{l}\text { Peak } \\
\#\end{array}$ & Systemic name & $\begin{array}{l}\text { Molecular } \\
\text { formula }\end{array}$ & $\begin{array}{l}\text { Mol. } \\
\text { wt. }\end{array}$ & $\begin{array}{l}t_{\mathrm{R}} \\
(\min )\end{array}$ & Conc. & $\begin{array}{l}\text { Characteristic mass fragments, } \\
\mathrm{m} / \mathrm{z} \text { (rel.\% BP) }\end{array}$ & $\begin{array}{l}\text { Area } \\
\text { Sum } \\
\text { (\%) }\end{array}$ \\
\hline & & & & & & $73(32), 83(32), 29(25), 56(22), 60(19)$ & \\
\hline 27. & $\begin{array}{l}\text { Hexanoic acid, 2-ethyl-, oxybis (2,1-ethanediyloxy-2,1- } \\
\text { ethanediyl) ester }\end{array}$ & $\mathrm{C}_{24} \mathrm{H}_{46} \mathrm{O}_{7}$ & 446 & 36.478 & 0.267 & $\begin{array}{l}\text { 171(99), 7(63), 127(45), 99(32), 87(30), } \\
55(21), 73(21), 43(18), 114(17), 172(14)\end{array}$ & 0.267 \\
\hline 28. & E-9-Methyl-8-tridecen-2-ol, acetate & $\mathrm{C}_{16} \mathrm{H}_{30} \mathrm{O}_{2}$ & 254 & 36.586 & 0.037 & $\begin{array}{l}43(99), 55(57), 97(31), 41(29), 109(17) \\
69(15), 71(14), 123(12), 112(11), 57(11)\end{array}$ & 0.037 \\
\hline 29. & $\begin{array}{l}\text { Cyclohexanol, 2-methyl-3-(1-methylethenyl)-, acetate, } \\
(1 a, 2 a, 3 a)-\end{array}$ & $\mathrm{C}_{12} \mathrm{H}_{20} \mathrm{O}_{2}$ & 196 & 38.058 & 0.065 & $\begin{array}{l}43(99), 136(24), 93(18), 121(18), 41(17) \\
55(13), 107(12), 95(12), 81(12), 94(10)\end{array}$ & 0.065 \\
\hline 30. & Tert-Hexadecanethiol & $\mathrm{C}_{16} \mathrm{H}_{34} \mathrm{~S}$ & 258 & 38.515 & 0.067 & $\begin{array}{l}57(99), 69(76), 43(71), 71(68), 55(61) \\
83(57), 41(54), 85(47), 97(35), 70(28)\end{array}$ & 0.067 \\
\hline 31. & Methyl-2-methoxyoct-2-enoate & $\mathrm{C}_{10} \mathrm{H}_{18} \mathrm{O}_{3}$ & 186 & 39.112 & 0.082 & $\begin{array}{l}43(99), 41(47), 115(44), 55(38), 85(36) \\
45(34), 69(27), 109(25), 29(24), 42(21)\end{array}$ & 0.082 \\
\hline 32. & 7-(1-Hydroxy-cyclohexyl)-2,2-dimethyl-hept-5-en-3-one & $\mathrm{C}_{15} \mathrm{H}_{26} \mathrm{O}_{2}$ & 238 & 41.82 & 0.202 & $\begin{array}{l}57(99), 85(44), 99(40), 81(35), 41(26) \\
55(21), 29(17), 83(14), 86(11), 43(10)\end{array}$ & 0.202 \\
\hline 33. & Tetradecanoic acid & $\mathrm{C}_{14} \mathrm{H}_{28} \mathrm{O}_{2}$ & 228 & 43.242 & 0.046 & $\begin{array}{l}73(99), 60(84), 43(68), 57(60), 41(46) \\
55(45), 129(37), 71(33), 69(28), 85(24)\end{array}$ & 0.046 \\
\hline 34. & Phen-1,4-diol, 2,3-dimethyl-5-trifluoromethyl- & $\mathrm{C}_{9} \mathrm{H}_{9} \mathrm{~F}_{3} \mathrm{O}_{2}$ & 206 & 43.987 & 0.105 & $\begin{array}{l}149(99), 83(29), 43(27), 151(26), 57(24) \\
55(23), 69(16), 71(15), 77(14), 41(13)\end{array}$ & 0.105 \\
\hline 35. & Estra-1,3,5(10)-trien-17ß-ol & $\mathrm{C}_{18} \mathrm{H}_{24} \mathrm{O}$ & 256 & 44.366 & 0.071 & $\begin{array}{l}43(99), 57(99), 55(71), 41(55), 73(47) \\
71(44), 45(39), 85(35), 83(35), 256(34)\end{array}$ & 0.071 \\
\hline 36. & 2-Pentadecanone, 6,10,14-trimethyl- & $\mathrm{C}_{18} \mathrm{H}_{36} \mathrm{O}$ & 268 & 44.936 & 0.077 & $\begin{array}{l}43(99), 58(89), 71(45), 57(42), 59(40), \\
41(37), 55(34), 69(24), 85(22), 95(20)\end{array}$ & 0.077 \\
\hline 37. & Pentadecanoic acid & $\mathrm{C}_{15} \mathrm{H}_{30} \mathrm{O}_{2}$ & 242 & 45.054 & 0.061 & $\begin{array}{l}\text { 73(99), 60(87), 43(86), 57(81), 41(73), } \\
55(64), 71(49), 129(45), 69(40), 29(36)\end{array}$ & 0.061 \\
\hline 38. & Pentadecanoic acid & $\mathrm{C}_{15} \mathrm{H}_{30} \mathrm{O}_{2}$ & 242 & 45.987 & 0.091 & $\begin{array}{l}73(99), 60(87), 43(86), 57(81), 41(73) \\
55(64), 71(49), 129(45), 69(40), 29(36)\end{array}$ & 0.091 \\
\hline 39. & 1-Hexadecanol, 2-methyl- & $\mathrm{C}_{17} \mathrm{H}_{36} \mathrm{O}$ & 256 & 46.126 & 0.073 & $\begin{array}{l}57(99), 43(88), 55(67), 41(63), 69(58) \\
56(57), 71(51), 83(46), 97(38), 70(34)\end{array}$ & 0.073 \\
\hline 40. & $\begin{array}{l}\text { 2-Butenal, 2-methyl-4-(2,6,6-trimethyl-1-cyclohexen-1- } \\
\text { yl)- }\end{array}$ & $\mathrm{C}_{14} \mathrm{H}_{22} \mathrm{O}$ & 206 & 46.683 & 0.077 & $\begin{array}{l}107(99), 123(96), 41(72), 206(72), 95(70) \\
81(64), 55(64), 191(63), 121(59), 135(59)\end{array}$ & 0.077 \\
\hline 41. & Z-(13,14-Epoxy)tetradec-11-en-1-ol acetate & $\mathrm{C}_{16} \mathrm{H}_{28} \mathrm{O}_{3}$ & 268 & 47.671 & 0.077 & $\begin{array}{l}43(99), 97(48), 69(48), 55(44), 41(36) \\
82(30), 67(29), 81(29), 84(27), 83(27)\end{array}$ & 0.077 \\
\hline 42. & n-Hexadecanoic acid & $\mathrm{C}_{16} \mathrm{H}_{32} \mathrm{O}_{2}$ & 256 & 53.368 & 0.496 & $\begin{array}{l}\text { 60(99),73(98), 57(84), 43(81), 55(76), } \\
41(57), 129(43), 71(37), 69(35), 83(26)\end{array}$ & 0.496 \\
\hline 43. & cis-13-Octadecenoic acid & $\mathrm{C}_{18} \mathrm{H}_{34} \mathrm{O}_{2}$ & 282 & 62.93 & 0.436 & $\begin{array}{l}55(99), 69(54), 41(46), 83(43), 97(39), \\
84(24), 43(24), 56(24), 67(22), 96(21)\end{array}$ & 0.436 \\
\hline 44. & 6-Octadecenoic acid & $\mathrm{C}_{18} \mathrm{H}_{34} \mathrm{O}_{2}$ & 282 & 63.897 & 0.244 & $\begin{array}{l}\text { 55(99), 97(85), 83(82), 69(75), 84(64), } \\
96(62), 81(61), 57(54), 264(49), 67(48)\end{array}$ & 0.244 \\
\hline 45. & 7-Methyl-Z-tetradecen-1-ol acetate & $\mathrm{C}_{17} \mathrm{H}_{32} \mathrm{O}_{2}$ & 268 & 69.535 & 0.076 & $\begin{array}{l}43(99), 55(66), 41(50), 81(26), 57(24) \\
85(23), 67(22), 69(22), 99(21), 83(20)\end{array}$ & 0.076 \\
\hline 46. & Z-5-Methyl-6-heneicosen-11-one & $\mathrm{C}_{22} \mathrm{H}_{42} \mathrm{O}$ & 322 & 70.771 & 0.085 & $\begin{array}{l}43(99), 55(87), 57(81), 41(75), 71(57) \\
83(47), 169(44), 85(43), 81(40), 69(40)\end{array}$ & 0.085 \\
\hline 47. & Hexadecanoic acid, 3-[(trimethylsilyl) oxy]propyl ester & $\mathrm{C}_{22} \mathrm{H}_{46} \mathrm{O}_{3} \mathrm{Si}$ & 386 & 72.529 & 0.058 & $\begin{array}{l}57(99), 43(86), 239(76), 73(68), 71(60) \\
130(52), 55(50), 41(48), 75(46), 85(38)\end{array}$ & 0.058 \\
\hline 48. & Heptacosane & $\mathrm{C}_{27} \mathrm{H}_{56}$ & 380 & 72.942 & 0.051 & $\begin{array}{l}57(99), 43(79), 71(62), 85(41), 55(28), \\
41(27), 69(17), 99(14), 56(13), 29(13)\end{array}$ & 0.051 \\
\hline 49. & 1,2-Benzenedicarboxylic acid, diisooctyl ester & $\mathrm{C}_{24} \mathrm{H}_{38} \mathrm{O}_{4}$ & 390 & 73.918 & 0.06 & $\begin{array}{l}\text { 149(99), 167(35), 57(34), 70(26), 41(22), } \\
71(22), 55(21), 43(20), 150(10), 83(10)\end{array}$ & 0.06 \\
\hline 50. & Di-n-octyl phthalate & $\mathrm{C}_{24} \mathrm{H}_{38} \mathrm{O}_{4}$ & 390 & 74.505 & 0.05 & $\begin{array}{l}\text { 149(99), 167(54), 57(44), 71(35), 70(28), } \\
43(27), 279(21), 41(21), 113(20), 55(16)\end{array}$ & 0.05 \\
\hline 51. & 1,3,2-Dioxaborolane, 4,4'-(1,4-butanediyl)bis[2-ethyl- & $\mathrm{C}_{12} \mathrm{H}_{24} \mathrm{~B}_{2} \mathrm{O}_{4}$ & 254 & 79.581 & 0.049 & $\begin{array}{l}\text { 99(99), 98(32), 43(27), 41(17), 57(17), } \\
83(13), 138(12), 29(11), 42(10), 55(10)\end{array}$ & 0.049 \\
\hline
\end{tabular}




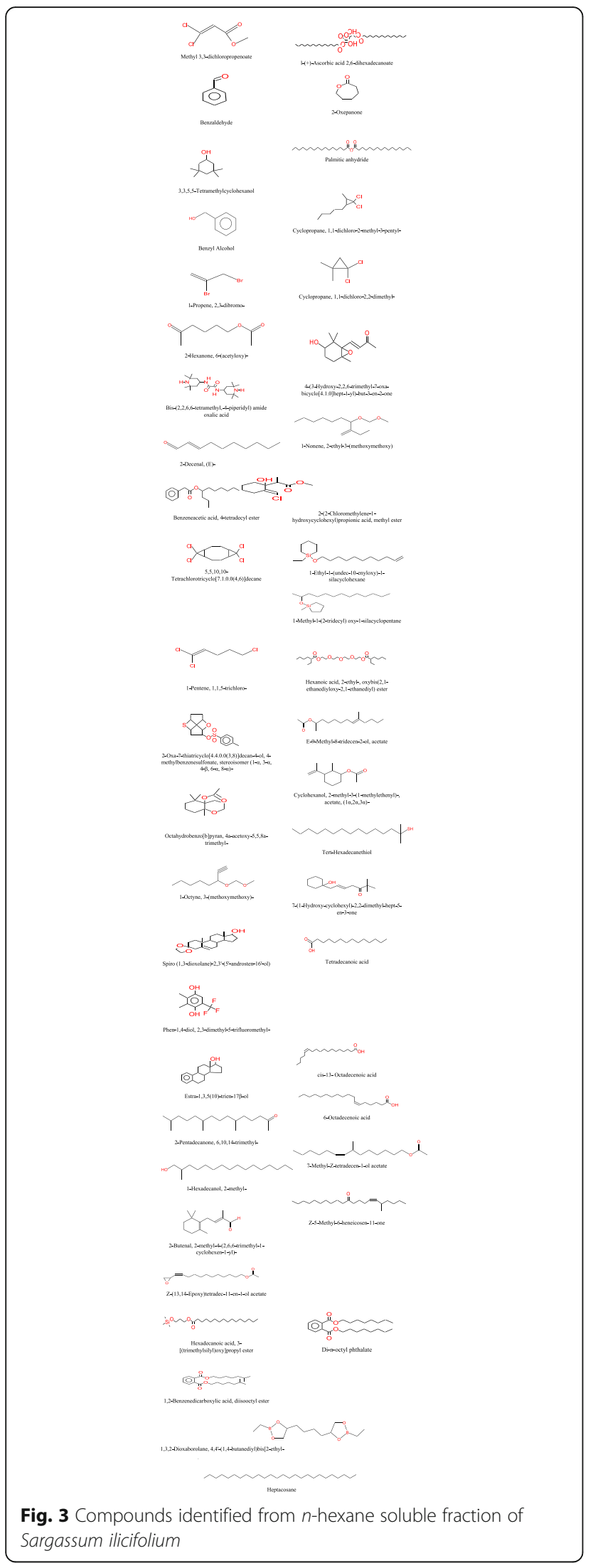

rats. The present findings further investigated and confirmed the hepatoprotective and nephroprotective role of $n$-hexane fraction of S.ilicifolium.

In the biological system oxidative stress may result in increased lipid peroxidation thus indicating cellular damage [30]. Excess production of free radicals may damage cell membranes and lipoproteins by a process called lipid peroxidation, resulting in the production of malondialdehyde (MDA). MDA is an end product of membrane damage, can bind with thiobarbituric acid, hence also called thiobarbituric acid reactive substances (TBARS). Glutathione can help in reducing the free radicals [31]. A decrease in GSH and increase in MDA is considered as a sign of liver dysfunction [3]. Reduction in GSH level of kidneys and lungs was reported by Taye and Abdel-Raheem [32], along with $\mathrm{CCl}_{4}$ intoxication. Ohta et al. [33] also reported GSH reduction in various organs of rats injected with $\mathrm{CCl}_{4}$. In this study glutathione (GSH) was improved and TBARS (MDA) was decreased in rats pretreated with $n$-hexane and methanol fractions of S. ilicifolium in AAP intoxicated rats as compared to AAP control group.

The hepatoprotective activity of different phytoconstituents like flavonoides, triterpenes, saponins and alkaloids and fatty acids has been reported earlier [31, 34]. In present study GC-MS analysis of $n$-hexane extract showed that hexadecanoic acid was found in highest concentration followed by cis-13- octadecenoic acid, hexanoic acid 2-ethyl-oxybis (2,1-ethanediyloxy-2,1ethanediyl) ester, 6-octadecenoic acid respectively. The fatty acids related compounds present in Sargassum fulvellum and $S$. thunbergii possess anti-inflammatory activities, as these are competitive inhibitors of cyclooxygenase and/or lipoxygenase, hence decrease the production of prostaglandins and leukotrienes [35, 36]. Biological activities of fatty acids from a brown alga Spatoglossum asperum has been reported earlier [37]. Hepatoprotective potential of $S$. ilicifolium may be due to presence of fatty acids that have been reported to ameliorate liver function enzymes in rats, which ultimately lead to reduced liver necrosis and inflammations [29, 38]. The $n$-hexadecanoic acid is known as an antiinflammatory compound [34]. Seaweeds also contain diterpenes, triterpenes and halogenated compounds with diverse biological activities as antibacterial, antioxidant, insecticidal and cytotoxic activities [39-41]. In this study, besides fatty acids, hydrocarbons, alcohols, aliphatic compounds, benzene derivatives, aldehyde and terpenoid were also found in $n$-hexane fraction of S.ilicifolium. Hepatoprotective activity of Silicifolium might be due to the presence of individual compounds or combinations of more than one compound. Like other natural products, presumably, seaweed acts as an antioxidant agent, increasing intracellular concentration 
of glutathione [42, 43]. It may enhance protein synthesis and regeneration of liver cells [44]. Polysaccharide from Sargassum sp., has been reported to decrease the MDA and increased glutathione in acetaminophen intoxicated rats [3].

\section{Conclusion}

Present study described that $n$-hexane and methanol soluble fraction of a brown seaweed S. ilicifolium, exhibited hepatoprotective activity via reducing liver marker enzymes and enhancing hepatic antioxidant level. Characterization of $n$-hexane soluble fraction of S. ilicifolium confirmed the presence of different volatile compounds, in which fatty acids were found to be in highest concentration followed by halogenated hydrocarbons, fatty acid derivatives and sterols. Further investigation is needed to examine the hepatoprotective effect of individual compounds identified from $n$-hexane soluble fraction of S. ilicifolium.

\section{Abbreviations}

LDH: Lactate dehydrogenase; AST: Aspartate aminotransferases; ALP: Alkaline phosphatase; ALT: Alanine aminotransferases; GC-MS: Gas chromatographymass spectrometry; S. ilicifolium: Sargassum ilicifolium; AAP: Acetaminophen; TBARS: Thiobarbituric acid reactive substances; GSH: Glutathione; MDA: Malondialdehyde

\section{Supplementary Information}

The online version contains supplementary material available at https://doi. org/10.1186/s40816-021-00274-4.

Additional file 1 Figure S1. Concentration of different compounds in n-hexane soluble fraction of Sargassum ilicifolium. Figure $\mathbf{S 2}$.

Concentration of different compounds in $n$-hexane soluble fraction of Sargassum ilicifolium (expansion of S-1). Figure S3. Concentration of different compounds in $n$-hexane soluble fraction of Sargassum ilicifolium (expansion of S-1). Figure S4. Concentration of different compounds in $n$-hexane soluble fraction of Sargassum ilicifolium (expansion of S-1). (DOCX $129 \mathrm{~kb}$ )

\section{Acknowledgements}

Support of Prof. Dr. Viqar Uddin Ahmad (now late), H.E.J. Research Institute of Chemistry, University of Karachi for GC-MS analysis and Dr. Aisha Begum, Department of Botany, University of Karachi for seaweed identification are acknowledged.

\section{Authors' contributions}

$\mathrm{KH} \& \mathrm{JA}$ conceived and designed the study. $\mathrm{KH} \& \mathrm{NS}$ performed experimental work, analyzed and interpreted the results. GC-MS profiling was done by HF \& MA. KH wrote the manuscript. SE \& JA improved the manuscript. All authors read the final manuscript.

\section{Funding}

No funding was provided.

\section{Declarations}

Ethics approval and consent to participate

The experiment was conducted according to the rules of Institutional Animal Ethics Committee (IAEC)/ Board of Advanced Studies and Research (BASR/ No./0584/Sc. dated 06-08-2010), University of Karachi.

\section{Consent for publication}

Not Applicable.

\section{Competing interests}

The authors declare that they have no competing interests.

\section{Author details}

${ }^{1}$ Institute of Biomedical Sciences, Dow University of Health Sciences, Karachi 75270, Pakistan. ${ }^{2}$ Department of Botany, Agricultural Biotechnology \& Phytopathology Laboratory, University of Karachi, Karachi 75270, Pakistan. ${ }^{3}$ Department of Biochemistry, Biotechnology \& Drug Development Laboratory, University of Karachi, Karachi 75270, Pakistan. ${ }^{4}$ H.E.J. Research Institute of Chemistry, University of Karachi, Karachi 75270, Pakistan. ${ }^{5}$ Department of Food Science \& Technology, University of Karachi, Karachi 75270, Pakistan.

Received: 17 July 2020 Accepted: 6 April 2021

Published online: 20 April 2021

\section{References}

1. Jaeschke H, Williams CD, McGill MR, Xie Y, Ramachandran A. Models of drug-induced liver injury for evaluation of phytotherapeutics and other natural products. Food Chem Toxicol. 2013;55:279-89. https://doi.org/10.101 6/j.fct.2012.12.063.

2. Hira K, Sultana V, Ara J, Ehteshamul-Haque S. Protective role of Sargassum species in liver and kidney dysfunctions and associated disorders in rats intoxicated with carbon tetrachloride and acetaminophen. Pak J Pharm Sci. 2017a;30:721-8

3. Hira K, Sultana V, Khatoon N, Ara J, Ehteshamul-Haque S. Protective effect of crude sulphated polysaccharides from Sargassum Swartzii (turn.) C.Ag. Against acetaminophen induced liver toxicity in rats. Clin Phytosci. 2019; https://doi.org/10.1186/s40816-019-0108-0.

4. Larson AM, Polson J, Fontana RJ, Davern TJ, Lalani E, Hynan LS, et al. Acetaminophen induced acute liver failure: results of a United States multicenter, prospective study. J Hepatol. 2005;42:1364-72. https://doi.org/1 0.1002/hep.20948.

5. Sohail N, Hira K, Tariq A, Sultana V, Ehteshamul-Haque S. Marine macroalgae attenuates nephrotoxicity and hepatotoxicity 6 induced by cisplatin and acetaminophen in rats. Environ Sci Pollut Res. 2019;26:25301-11. https://doi.org/10.1007/s11356-019-05704-y.

6. Hong M, Li S, Tan HY, Wang N, Tsao N-W, Feng Y. Current status of herbal medicines in chronic liver disease therapy: the biological effects, molecular targets and future prospects. Int J Mol Sci. 2015;16:28705-45. https://doi. org/10.3390/ijms161226126.

7. Blunden G. Marine algae as sources of biologically active compounds. Inter discipl Sci Rev. 1993;18:73-80. https://doi.org/10.1179/isr.1993.18.1.73.

8. Ruqqia K, Sultana V, Ara J, Ehteshamul-Haque S, Athar M. Hypolipidaemic potential of seaweeds in normal, triton-induced and high fat diet- induced hyperlipidaemic rats. J Appl Phycol. 2015;27:571-9. https://doi.org/10.1007/ s10811-014-0321-7.

9. Akhtar P, Ambreen HK, Sultana V, Ara J, Ehteshamul-Haque S. Hypoglycemic potential of some seaweeds from Karachi coast of Pakistan. Pak J Pharm Sci. 2019;32:1599-05.

10. Smit AJ. Medicinal and pharmaceutical uses of seaweed natural products: a review. J Appl Phycol. 2004;16:245-62. https://doi.org/10.1023/B:JAPH. 0000047783.36600.ef.

11. Ang PO. Phenology of Sargassum spp. in Tung ping Chau marine park, Hong Kong SAR, China. J Appl Phycol. 2006;18:629-36. https://doi.org/10.1 007/s10811-006-9071-5.

12. Zhang QS, Li W, Pan JH. Size-dependence of reproductive allocation of Sargassum thunbergii (Sargassaceae, Phaeophyta) in Bohai Bay, China. Aquat Bot. 2009;91:194-8. https://doi.org/10.1016/j.aquabot.2009.06.003.

13. Quintal-Novelo C, Rangel-Méndez J, Ortiz-Tello Á, Graniel-Sabido M, Vaca RPCD, Moo-Puc R. A Sargassum fluitans Borgesen ethanol extract exhibits a 
hepatoprotective effect in-vivo in acute and chronic liver damage models. Biomed Res Int. 2018. https://doi.org/10.1155/2018/6921845.

14. Yende SR, Harle UN, Chaugule BB. Therapeutic potential and health benefits of Sargassum species. Pharmacogn Rev. 2014;8:1-7. https://doi.org/10.4103/ 0973-7847.125514

15. Chandraraj S, Prakash B, Navanath K. Immunomodulatory activities of ethyl acetate extracts of two marine sponges Gelliodes fibrosa and Tedania anhelans and brown algae Sargassum ilicifolium with reference to phagocytosis. Res J Pharm Biol Chem Sci. 2010;1:302-7.

16. Rebecca LJ, Dhanalakshmi V, Shelhar C. Antibacterial activity of Sargassum ilicifolium and Kappaphycus alvarezii. J Chem Pharm Res. 2012;4:700-5.

17. Selvarani T, Prabhu BK, Thenmozhi K. Effect of aqueous extract from the seaweeds, Sargassum ilicifolium on three types of non-pathogenic terrestrial bacteria. Int J Med Arom Plants. 2013;3:169-77.

18. Ambreen R, Hira K, Tariq A, Sultana V, Ara J. Evaluation of biochemical component and antimicrobial activity of some seaweed occurring at Karachi Coast. Pak J Bot. 2012;44:1799-03.

19. Dar A, Baig HS, Saifullah SM, Ahmad VU, Yasmeen S, Nizamuddin M. Effect of seasonal variation on the anti-inflammatory activity of Sargassum wightii growing on the N. Arabian Sea coast of Pakistan. J Experiment Mar Biol Ecol. 2007;351:1-9. https://doi.org/10.1016/j.jembe.2007.03.019.

20. Hira K, Tariq RM, Sultana V, Ara J, Ehteshamul-Haque S. Effect of seaweeds occurring at Karachi coast on mosquito larvae and liver function in rats. Pak J Pharm Sci. 2017b;30:387-91.

21. National Research Council (US). Subcommittee on laboratory animal nutrition. Nutrient requirements of laboratory animals: $4^{\text {th }}$ ed. Washington (DC): National Academies Press (US); 1995

22. Samarth RM, Panwar M, Kumar M, Soni A, Kumar M, Kumar A. Evaluation of antioxidant and radical-scavenging activities of certain radioprotective plant extracts. Food Chem. 2008;106:868-73. https://doi.org/10.1016/j.foodchem.2 007.05.005.

23. Ohkawa H, Ohishi N, Yagi K. Assay for lipid peroxides in animal tissues by the thiobarbitoric acid reaction. Anal Biochem. 1979;95:351-8. https://doi. org/10.1016/0003-2697(79)90738-3.

24. Farhat, H., Urooj F, Tariq A, Sultana V, Ansari M, Ahmad VU, EhteshamulHaque S Evaluation of antimicrobial potential of endophytic fungi associated with healthy plants and characterization of compounds produced by endophytic Cephalosporium and Fusarium solani Biocatal Agric Biotechnol 2019; doi.https://doi.org/10.1016/j.bcab.2019.101043, 18, 101043.

25. Armitage P, Berry G. Statistical methods in medical research. 3rd ed. Oxford: Blackwell Science; 1994.

26. Raghavendran HB, Sathivel A, Yogeeta RSSK, Devaki T. Efficacy of Sargassum polycystum (Phaeophyceae) sulphated polysaccharide against paracetamolinduced DNA fragmentation and modulation of membrane-bound phosphatases during toxic hepatitis. Clin Exp Pharmacol Physiol. 2007;34: 142-7. https://doi.org/10.1111/j.1440-1681.2007.04539.x.

27. Raghavendran $\mathrm{HB}$, Srinivasan P. Effect of crude sulphated polysaccharide from brown algae against acetaminophen-induced toxicity in rats. Can J Physiol Pharmacol. 2008;86:660-6. https://doi.org/10.1139/Y08-072.

28. Yousef MI, Omar SA, El-Guendi MI, Abdelmegid LA. Potential protective effects of quercetin and curcumin on paracetamol-induced histological changes, oxidative stress, impaired liver and kidney functions and hepatotoxicity in the rat. Food Chem Toxicol. 2010;48:3246-61. https://doi. org/10.1016/j.fct.2010.08.034.

29. Taj D, Tariq A, Sultana V. Protective role of Stokeyia indica in liver dysfunction and associated complications in acetaminophen intoxicated rats. Clin Phytosci. 2019; https://doi.org/10.1186/s40816-019-0122-2.

30. Somasundaram A, Karthikeyan R, Velmurugan V, Dhandapani B, Raja M. Evaluation of hepatoprotective activity of Kyllinga nemoralis (Hutch \& Dalz) rhizomes. J Ethnopharmacol. 2010;127:555-7. https://doi.org/10.1016/j.jep.2 009.11.014.

31. Kumar A, Rai N, Kumar N, Gautam P, Kumar JS. Mechanism involved in hepatoprotection of different herbal products. Int J Res Pharm Sci. 2013;4: 112-7.

32. Taye A, Abdel-Raheem IT. Hepatoprotective effect of the selective mineralocorticoid receptor antagonist, eplerenone against carbon tetrachloride induced liver injury in rats. Ann Hepatol. 2012;11:384-91. https://doi.org/10.1016/S1665-2681(19)30935-4.

33. Ohta $Y$, Kongo $M$, Sasaki E. Therapeutic effect of melatonin on carbon tetrachloride-induced acute liver injury in rats. J Pineal Res. 2000;28:119-26. https://doi.org/10.1034/j.1600-079X.2001.280208.x.
34. Aparna V, Dileep KV, Mandal PK, Karthe P, Sadasivan C, Haridas M. Antiinflammatory property of n-hexadecanoic acid: structural evidence and kinetic assessment. Chem Biol Drug Des. 2012;80:434-9. https://doi.org/1 0.1111/j.1747-0285.2012.01418.x.

35. James MJ, Gibson RA, Cleland LG. Dietary polyunsaturated fatty acids and inflammatory mediator production. Am J Clin Nutr. 2000;71:343-8.

36. Kang JY, Khan MNA, Park NH, Cho JY, Lee MC, Fujii H, et al. Antipyretic, analgesic and anti-inflammatory activities of seaweed Sargassum fulvellum and Sargassum thunbergii in mice. J Ethnopharmacol. 2008;116:187-90. https://doi.org/10.1016/j.jep.2007.10.032

37. Ara J, Sultana V, Qasim R, Ehteshamul-Haque S, Ahmad VU. Biological activity of Spatoglossum asperum: a brown alga. Phytother Res. 2005;19(7): 618-23. https://doi.org/10.1002/ptr.1699.

38. Al Tuwaijri A, Akdamar K, Diluzio R. Modification of galactosamine-induced liver injury in rats by reticulo-endothelial stimulation or depression. Hepatology. 1981;1:107-13. https://doi.org/10.1002/hep.1840010204.

39. Shui-Chun MA, Yue-Wei GU. Sesquiterpenes from Chinese red alga Laurencia okamurai. Chin J Nat Med. 2010;8:321-5.

40. Gressler V, Stein ÉM, Dörr F, Fujii MT, Colepicolo P, Pinto E. Sesquiterpenes from the essential oil of Laurencia dendroidea (Ceramiales, Rhodophyta): isolation, biological activities and distribution among seaweeds. Rev Bras. 2011:21:248-54. https://doi.org/10.1590/S0102-695X2011005000059.

41. Horincar VB, Parfene G, Tyagi AK, Gottardi D, Dinică R, Guerzoni ME, et al. Extraction and characterization of volatile compounds and fatty acids from red and green macroalgae from the Romanian Black Sea in order to obtain valuable bio-additives and biopreservatives. J Appl Phycol. 2014;26:551-9. https://doi.org/10.1007/s10811-013-0053-0.

42. Tariq A, Ara J, Sultana V, Ehteshamul-Haque S, Athar M. Antioxidant potential of seaweeds occurring at Karachi coast of Pakistan. J Appl Bot Food Qual. 2011;84:207-12

43. El-Sohafy SM, Alqasoumi SI, Metwally AM, Omar AA, Amer MM, Abou Shoer MI. Evaluation of the hepatoprotective activity of some plants belonging to the tribe Cynareae growing in Egypt. J Med Plants Res. 2013;7:324-8.

44. Saller R, Melzer J, Rechling J, Brignoli R, Meier R. An updated systematic review of the pharmacology of silymarin. Forsch Komplementarmed. 2007; 14(2):70-80. https://doi.org/10.1159/000100581.

\section{Publisher's Note}

Springer Nature remains neutral with regard to jurisdictional claims in published maps and institutional affiliations.

\section{Submit your manuscript to a SpringerOpen ${ }^{\circ}$ journal and benefit from:}

- Convenient online submission

- Rigorous peer review

- Open access: articles freely available online

High visibility within the field

- Retaining the copyright to your article

Submit your next manuscript at $>$ springeropen.com 